\title{
CME Cardiology (100622): self-assessment questionnaire
}

\author{
Edited by Clifford Garratt and Tahseen A Chowdhury
}

\section{SAQs and answers are ONLINE for RCP fellows and collegiate members}

The SAQs printed in the CME section can only be answered online to achieve external CPD credits. Any comments should be sent in via email only: clinicalmedicine@rcplondon.ac.uk

\section{Format}

Candidates are asked to choose the best answer from the five possible answers. This best of five format is used in many medical examinations, however the questions are not intended to be representative of those used in the MRCP(UK) Part 1 or Part 2 Written Examinations.

\section{The answering process}

1 Go to www.rcplondon.ac.uk/SAQ

2 Log on using your usual RCP username and password

3 Select the relevant CME question paper

4 Answer all 10 questions by selecting the best answer from the options provided

5 Once you have answered all the questions, click on Submit

\section{Registering your external CPD credits}

Carrying out this activity allows you to claim two external CPD credits. These will be automatically transferred to your CPD diary, where you can review the activity and claim your points.

1 You are asked to assess the risk of sudden death and need for primary prevention implantable cardioverterdefibrillator (ICD) in a 65-year-old gentleman with a history of an ST elevation myocardial infarction 6 months prior. He is on maximal secondary prevention medication. $\mathrm{He}$ is asymptomatic for both angina and heart failure.

In addition to left ventricular systolic function on echocardiography, which of these risk prediction methods would be of most use in your assessment?

(a) elevated $\mathrm{N}$-terminal pro-B-type natriuretic peptide levels

(b) inducibility of monomorphic ventricular tachycardia during electrophysiological studies (c) left ventricular late gadolinium enhancement on contrast cardiac magnetic resonance imaging

(d) QRS duration $>120 \mathrm{~ms}$

(e) upsloping ascending early repolarisation.

2 In which of the following settings would genetic testing aid in your decision with regard to risk of future sudden death?

(a) an asymptomatic 56-year-old with Brugada syndrome

(b) a 23-year-old with syncope and an abnormal signal averaged electrocardiogram

(c) a 27-year-old survivor of an unspecified cardiac arrest

(d) a 44-year-old with hypertrophic cardiomyopathy and syncope

(e) none of the above.

3 Which of the following is an indication to refer a patient for atrial fibrillation (AF) ablation?

(a) as a curative treatment for a patient with long-standing persistent $\mathrm{AF}$

(b) as treatment for a symptomatic patient with paroxysmal $\mathrm{AF}$ who would prefer not to be on antiarrhythmic medications

(c) to improve the long-term prognosis of an asymptomatic patient with AF

(d) to improve the symptoms of a patient with obesity and obstructive sleep apnoea

(e) to reduce the stroke risk of a patient with $\mathrm{AF}$ who is unable to take oral anticoagulation medication.

4 A 54-year-old man complained of increasing shortness of breath 1 month following an AF ablation. His electrocardiogram showed sinus tachycardia. Which of the following tests should be organised urgently first?
(a) chest X-ray
(b) computerised tomography chest
(c) echocardiogram
(d) troponin
(e) 24-hour Holter

5 Which of the following statements about atrial fibrillation (AF) ablation is true?

(a) AF ablation exposes the patient to a significant amount of radiation.

(b) AF ablation should only be considered as a last resort in the management of patients with AF and should be delayed as long as possible. 
(c) A patient requiring cardioversion for $\mathrm{AF}$ after 3 days and has since remained in sinus rhythm has paroxysmal AF.

(d) Cryoballoon ablation for AF is time consuming.

(e) Pulmonary vein isolation is the cornerstone of any ablation strategy.

6 Which of the following statements regarding the prognostic impact of atrial fibrillation (AF) in heart failure (HF) is true?

(a) $\mathrm{AF}$ in $\mathrm{HF}$ with preserved ejection fraction (HFPEF) has an overall better prognosis than $\mathrm{AF}$ in $\mathrm{HF}$ with reduced ejection fraction (HFREF)

(b) AF in HFREF confers a greater risk of stroke compared with $A F$ in HFPEF

(c) $\mathrm{AF}$ is more likely to occur in patients with less severe $\mathrm{HF}$ symptoms

(d) Presence of AF is associated with increased risk of mortality in patients with $\mathrm{HF}$ irrespective to the left ventricular ejection fraction

(e) The negative prognostic impact of $\mathrm{AF}$ in $\mathrm{HF}$ is observed in patients with HFREF only and not in those with HFPEF.

7 Which of the following statements regarding treatment strategies of atrial fibrillation (AF) in heart failure (HF) is true?

(a) Calcium channel antagonists (such as verapamil) and betareceptor blockers (such as bisoprolol) are equally indicated for $\mathrm{AF}$ control in $\mathrm{HF}$ with reduced ejection fraction (HFREF).

(b) 'Pace and ablate' strategy should be used as a first-line option in HF patients with AF.

(c) Pulmonary vein isolation using catheter ablation obviates the need for anti-arrhythmic medication thus avoiding potential adverse effects.

(d) There is ample evidence that rhythm control is superior to rate control in $\mathrm{HF}$ patients with $\mathrm{AF}$.

(e) There is no particular need for measuring serum digoxin levels in patients with HF.

8 Prasugrel is not recommended in which of the following?
(a) age $\geq 75$ years
(b) body weight $<60 \mathrm{~kg}$
(c) previous history of cerebrovascular disease
(d) previous intracranial haemorrhage
(e) all of above

9 On discharge from hospital post ST segment elevation myocardial infarction, which combination of medications is commonly prescribed?

(a) aspirin, $\mathrm{P}_{2} \mathrm{Y}_{12}$ antagonist, atorvastatin $80 \mathrm{mg}$, angiotensinconverting-enzyme (ACE) inhibitor, beta blocker (b) aspirin, simvastatin $80 \mathrm{mg}$, ACE inhibitor, beta blocker, eplerenone

(c) aspirin, $\mathrm{P}_{2} \mathrm{Y}_{12}$ antagonist, atorvastatin $80 \mathrm{mg}$, diltiazem, beta blocker

(d) $\mathrm{P}_{2} \mathrm{Y}_{12}$ antagonist, spironolactone, atorvastatin $80 \mathrm{mg}$, beta blocker, ACE inhibitor

(e) aspirin, $P 2 Y_{12}$ antagonist, atorvastatin $80 \mathrm{mg}$, isosorbide mononitrate

10 A 56-year-old man presented with a 2-hour history of chest pain. The electrocardiogram (ECG) below was recorded on arrival by the paramedics.

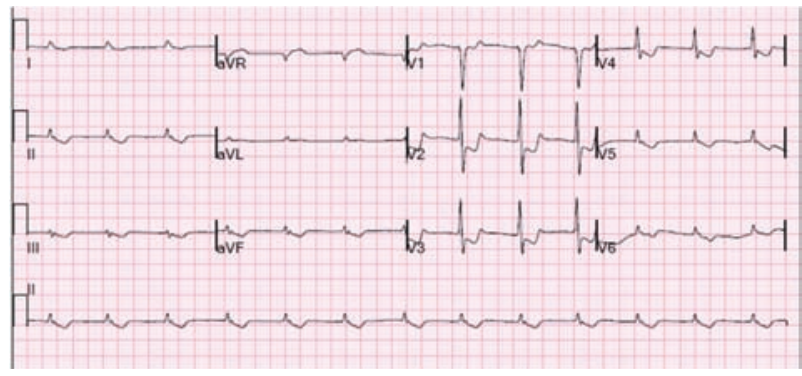

\section{Based on the history and ECG findings, which of the} following statements is correct?

(a) ECG shows an acute anterolateral ST segment elevation myocardial infarction (STEMI) and primary percutaneous coronary intervention (PPCI) is the treatment of choice.

(b) ECG shows an inferior STEMI and PPCI is the treatment of choice.

(c) ECG shows an inferior STEMI and thrombolysis is the treatment of choice.

(d) ECG shows a true posterior STEMI and PPCI is the treatment of choice.

(e) None of the above.

\section{CME Genitourinary medicine SAQ}

\section{Answers to the CME SAQ published in Clinical Medicine April 2016}

$\begin{array}{llllllllll}\text { Q1 } & \text { Q2 } & \text { Q3 } & \text { Q4 } & \text { Q5 } & \text { Q6 } & \text { Q7 } & \text { Q8 } & \text { Q9 } & \text { Q10 } \\ \text { (e) } & \text { (a) } & \text { (a) } & \text { (c) } & \text { (b) } & \text { (a) } & \text { (d) } & \text { (a) } & \text { (c) } & \text { (a) }\end{array}$

